\title{
Expression of mRNAs Encoding ARPP-16/19, ARPP-21, and DARPP-32 in Human Brain Tissue
}

\author{
Stefan Brené, ${ }^{1}$ Nils Lindefors, ${ }^{1}$ Michelle Ehrlich, ${ }^{3}$ Tanaquil Taubes, ${ }^{3}$ Atsuko Horiuchi, ${ }^{3}$ Jutta Kopp, ${ }^{1}$ Hâkan \\ Hall, ${ }^{1}$ Göran Sedvall, ${ }^{1}$ Paul Greengard, ${ }^{3}$ and Håkan Persson ${ }^{2, a}$ \\ 'Department of Clinical Neuroscience, and 'Department of Medical Chemistry, Laboratory of Molecular Neurobiology, \\ Karolinska Institutet, S-171 76 Stockholm, Sweden, and ' 2 Laboratory of Molecular and Cellular Neuroscience, Rockefeller \\ University, New York, New York
}

In this study we have isolated and sequenced human cDNAs for the phosphoproteins DARPP-32, ARPP-21, and ARPP-16/ 19 , and have compared these sequences to previously characterized bovine and rat cDNAs. In situ hybridization and Northern blot analysis with the human CDNA probes were used to study the expression of mRNAs encoding ARPP-16/ 19, ARPP-21, and DARPP-32 in human postmortem brain tissue. In situ hybridization was performed using horizontal whole hemisphere sections. Five representative levels of the brain ranging from $71 \mathrm{~mm}$ to $104 \mathrm{~mm}$ ventral to vertex were examined. All three probes showed distinct hybridization patterns in the caudate nucleus, putamen, nucleus accumbens, and the amygdaloid complex. For ARPP-16/19 mRNA, a hybridization signal comparable to the signal in caudate nucleus, putamen, and nucleus accumbens was also detected in the neocortex. ARPP-21 and DARPP-32 mRNA, on the other hand, were present in lower levels in neocortical regions. DARPP-32 mRNA was abundant in the cerebellar cortex at the level of the Purkinje cell layer. High levels of ARPP-16/19 and ARPP-21 mRNA were also found in the cerebellar cortex, where they were confined to deeper layers. The present result demonstrate that mRNAs for the three phosphoproteins are expressed in overlapping, but also distinct, areas of the human brain that in many cases coincide with previously described distribution of the dopamine $D_{1}$ receptor.

[Key words: cAMP, dopamine, human brain, in situ hybridization, phosphoprotein, mRNA]

A number of phosphoproteins have been shown to act as intracellular messengers in the mammalian CNS. Receptor-mediated phosphorylation and dephosphorylation of such proteins constitute important pathways for the regulation of neuronal function (Greengard, 1987). An extensively studied phosphoprotein is the dopamine and cAMP-regulated phosphoprotein

Received Jan. 27, 1993; revised July 26, 1993; accepted Aug. 16, 1993.

This work was supported by grants from the Swedish Medical Research Council (8653 and 3560), the National Institute of Mental Health (Grants MH-44814), the Swedish Society of Medicine, Åke Wibergs Stiftelse, and funds from the Karolinska Institute. S.B. was supported by a fellowship from the Swedish Medical Research Council.

Correspondence should be addressed to Nils Lindefors, M.D., Ph.D., Department of Psychiatry and Psychology, Karolinska Hospital, S-171 76 Stockholm, Sweden.

${ }^{a}$ Deceased, May 16, 1993.

Copyright @ 1994 Society for Neuroscience $0270-6474 / 94 / 140985-14 \$ 05.00 / 0$
DARPP-32 (Walaas et al., 1983). In the densely dopamine- and glutamate-innervated rat caudate-putamen (Fonnum et al., 1981; Björklund and Lindvall, 1984), DARPP-32 is expressed in medium-size spiny neurons (Ouimet and Greengard, 1990) that also express dopamine $D_{1}$ receptors (Walaas and Greengard, 1984). The function of DARPP-32 seems to be regulated by receptor stimulation. Thus, dopaminergic $\left(D_{1}\right)$ and glutamatergic (NMDA) receptor stimulation both regulate the extent of DARPP-32 phosphorylation but in opposite directions (Halpain et al., 1990). Dopamine $D_{1}$ receptor stimulation enhances cAMP formation resulting in the phosphorylation of DARPP-32 (Walaas and Greengard, 1984), and phosphorylated DARPP-32 is a potent protein phosphatase- 1 inhibitor (Hemmings et al., 1984). Glutamatergic NMDA receptor stimulation elevates intracellular calcium, which leads to an activation of calcineurin and dephosphorylation of phospho-DARPP-32, thereby reducing the phosphatase-1 inhibitory activity of DARPP-32 (Halpain et al., 1990).

Three additional cAMP-regulated phosphoproteins, ARPP16, ARPP-19, and ARPP-21, have been purified (Hemmings and Greengard 1989; Horiuchi et al., 1990). The primary structures of these phosphoproteins were first obtained from partial or complete amino acid sequencing and subsequently deduced in their entirety by nucleotide sequencing of bovine cDNA clones. Bovine DARPP-32, ARPP-16, ARPP-19, and ARPP-21 have apparent molecular weights on SDS-PAGE of about 32, 16, 19 , and $21 \mathrm{kDa}$, respectively. The amino acid sequences of ARPP16 and ARPP-19 are identical except that ARPP- 19 contains 16 additional amino-terminal amino acids. In contrast to DARPP-32, the functions of ARPP-16 and ARPP-21 are unknown, and there is no sequence homology between the three proteins (Kurihara et al., 1988, 1989; Horiuchi et al., 1990).

The regional distribution of these phosphoproteins has been described in rat brain using immunohistochemical techniques. DARPP-32 is most abundant in the caudate-putamen and nucleus accumbens, regions that receive a rich dopamine innervation from the mesencephalon. DARPP-32 is also found in dopamine innervated limbic structures and in the neocortex (Walaas et al., 1983; Ouimet et al., 1984). ARPP-16 and ARPP19 are present in several rat brain regions including caudateputamen, nucleus accumbens, olfactory tubercle, amygdaloid complex, globus pallidus, substantia nigra, hippocampus, thalamus, hypothalamus, and cerebral cortex (Girault et al., 1990). ARPP-19, but not ARPP-16, is present in the cerebellar cortex, midbrain structures, including the periaqueductal gray, pons, 
medulla, and the spinal cord (Girault et al., 1990). ARPP-21 is found in the caudate-putamen, globus pallidus, entopeduncular nucleus, substantia nigra pars reticulata, and in the dopamincinnervated limbic structures, nucleus accumbens, olfactory tubercle, the amygdaloid complex, and the cerebral cortex (Ouimet et al., 1989; Girault et al., 1990). Cells expressing DARPP$32 \mathrm{mRNA}$ in rat brain tissue have been described using a rat DARPP-32 mRNA-specific oligonucleotide for in situ hybridization analysis (Schalling et al., 1990). In the human brain the distribution patterns of mRNA for DARPP-32, ARPP-16/ ARPP-19, and ARPP-21 have previously not been described.

Here we report on the isolation and sequence analysis of cDNAs for human DARPP-32, ARPP-16/19, and ARPP-21. We have used these human cDNA clones as hybridization probes for whole hemisphere human tissue sections to analyze the regional distribution of DARPP-32, ARPP-16/19 (the cDNA probe used detects mRNAs for both ARPP-16 and ARPP-19), and ARPP-21 mRNAs. The results show that all three mRNAs are expressed in the caudate nucleus, putamen, nucleus accumbens, cerebellar cortex, and neocortex, although their relative levels in these areas differ.

\section{Materials and Methods}

Preparation of human brain tissue. The procedure for obtaining human postmortem tissue was approved by the ethics committee of the Karolinska Institute. Brain tissue was obtained from three subjects, 51, 64 , and 76 years of age, who had died from acute cardiorespiratory failure in all cases. The brains were acquired at clinical autopsy from the National Institute of Forensic Medicine at the Karolinska Institute, Stockholm, Sweden. The postmortem time varied from 3 to $22 \mathrm{hr}$. A complete clinical autopsy of all organs was performed. Specimens of blood, urine, liver, gastric contents, and kidney underwent toxicological screening for 218 neuroactive drugs including alcohol, antidepressants, antipsychotics, and minor tranquilizers. No such drugs were detected in the subjects from which the autopsy brain material was obtained. None of the brains showed any evidence of macroscopic pathology. For the subjects, medical records from all hospitals in the Stockholm region, as well as register data from the crime registers and social security files, were searched for and examined. There were no indices of substance abuse, psychiatric, or neurologic disease in any of the subjects.

After carefully dividing the brain in the midsagittal line, each hemisphere was frozen with the sagittal plane facing down on the glass plate. To avoid freeze drying, each hemisphere with the glass plate was placed in a plastic bag and the air was sucked out from the bag by a water pump before freezing $\left(-85^{\circ} \mathrm{C}\right)$ and storage for up to 4 years before further preparations.

In preparation for cryosectioning, the glass plate was removed from the frozen hemisphere and steel needles were introduced perpendicularly to the sagittal plane through the anterior and posterior commissure. These steel needles were used to define a horizontal section plane perpendicular to the intercommissural and sagittal plane, and to fixate the hemisphere in a metal frame mounted on a cooled $\left(-70^{\circ} \mathrm{C}\right)$ stage. It was embedded in a semiliquid gel $\left(4^{\circ} \mathrm{C}\right)$ of carboxymethylcellulose (CMC), which was frozen to a tissue $/ \mathrm{CMC}$ block at $-85^{\circ} \mathrm{C}$.

Serial sectioning of the brain/CMC block was performed with a heavyduty cryomicrotome (PMV400, LKB, Stockholm, Sweden). After equilibration over night to the temperature in the cryostat $\left(-17^{\circ} \mathrm{C}\right)$, the hemisphere was cut into $100-\mu \mathrm{m}$-thick slices for in situ hybridization. Some material from smaller blocks of tissue was also prepared for in situ hybridization in 14- $\mu$ m-thick sections. These were obtained by punching out regions of interest directly from the frozen hemisphere/ CMC block and stored at $-85^{\circ} \mathrm{C}$ until sectioning in a cryostat (Leitz, Wetzlar, Germany). The localization of the tissue section is presented as the distance $(\mathrm{mm})$ from vertex of the hemisphere in the vertical plane. Cortical Brodmann regions were defined according to a brain atlas (Nieuwenhuys et al., 1988).

Cloning and sequence analysis of human DARPP-32, ARPP-16/19, and $A R P P-21 c D N A s$. Fragments of the bovine and/or rat $c D N A s$ containing coding sequence for DARPP-32, ARPP-21, and ARPP-16/19 were isolated and labeled with $\alpha{ }^{32} \mathrm{P}-\mathrm{dCTP}$ by the random priming method. These probes were used to screen an oligo-dT-primed human caudate cDNA library in $\lambda g t 11$ (American Tissue Culture Collection) and an oligo-dT- and random-primed library in $\lambda$ ZAPII (kindly provided by Dr. Karen O'Malley) by standard methods (Maniatis et al., 1989). Isolated clones were restriction mapped, and fragments containing coding sequences identified by Southern blotting. These fragments were either subcloned into M13 or directly sequenced in both directions from the rescued (according to the protocol provided by Stratagene) Bluescript plasmid by the Sanger dideoxy method with modified T7 polymerase (Sequenase 2.0, U.S. Biochemical) using the M13 universal primer, T3 or T7 primers, or oligonucleotide primers based on previous sequence.

Preparation of human brain tissues and RNA and Northern blot analysis. Dissected brain tissue was homogenized in $4 \mathrm{M}$ guanidine isothiocyanate, $0.1 \mathrm{M} \beta$-mercaptoethanol, $0.025 \mathrm{M}$ sodium citrate, $\mathrm{pH} 7.0$, and the RNA was purified on a cesium chloride gradient (Maniatis et al., 1989). Polyadenylated RNA was isolated by oligo-dT-cellulose chromatography and spectrophotometrically quantified. Fifteen-microgram samples were size fractionated on $1 \%$ agarose, $0.7 \%$ formaldehyde gels, and transferred to Hybond-C-Extra membranes according to the protocol provided by the manufacturer (Amersham, Buckinghamshire, England). The filters were successively hybridized to nick-translated probes (specific activity of approximately $10^{9} \mathrm{cpm} / \mu \mathrm{g}$ ) derived from the human clones. The filters were washed at high stringency $[0.1 \times$ saline-sodium citrate (SSC), $0.1 \%$ SDS at $\left.54^{\circ} \mathrm{C}\right]$ and exposed to XAR-5 Kodak film for $10-15 \mathrm{~d}$.

In situ hybridization. Tissue sections, $100 \mu \mathrm{m}$ thick (hemispheres) or $14 \mu \mathrm{m}$ thick (tissue blocks), were examined. The sections were thawed onto poly-L-lysine-coated slides $(50 \mu \mathrm{g} / \mathrm{ml})$. The tissues were fixed in $10 \%$ formalin in phosphate-buffered saline for $30 \mathrm{~min}$, rinsed twice for $4 \mathrm{~min}$ in PBS, and delipidated in a graded series of ethanol, including a $5 \mathrm{~min}$ incubation with chloroform. The sections were then air dried. The same human cDNA fragments as for the Northern blot experiments were labeled with $\alpha{ }^{-35} \mathrm{~S}-\mathrm{dATP}$ by nick translation, to a specific activity of approximately $5 \times 10^{8} \mathrm{cpm} / \mu \mathrm{g}$. The labeled probes were then separated from unincorporated nucleotides on a Sephadex G-50 column and $5 \times 10^{6} \mathrm{cpm}$ of probe was added per milliliter of hybridization cocktail. The hybridization cocktail contained $50 \%$ formamide, $4 \times$ SSC $(1 \times \mathrm{SSC}=0.15 \mathrm{~m} \mathrm{NaCl}, 0.015 \mathrm{~m}$ sodium citrate $), 1 \times$ Denhardt's solution, $1 \%$ Sarcosyl, $0.02 \mathrm{M} \mathrm{Na}_{3} \mathrm{PO}_{4}$ (pII 7.0), $10 \%$ dextran sulfate, $0.5 \mathrm{mg} / \mathrm{ml}$ yeast tRNA, $0.06 \mathrm{M}$ dithiothreitol, and $0.1 \mathrm{mg} / \mathrm{ml}$ sheared salmon sperm DNA. Each whole hemisphere section was incubated with $2.0 \mathrm{ml}$ of hybridization cocktail, and each tissue block section was incubated with $0.1 \mathrm{ml}$ of hybridization cocktail containing the labeled probe. All sections were covered by parafilm as coverslips during the hybridization in a humidified chamber. Hybridization was performed for $18 \mathrm{hr}$ at $42^{\circ} \mathrm{C}$. As a hybridization control, the pBSKS plasmid (Stratagene, La Jolla, CA) without insert was labeled with $\alpha{ }^{-35}$ S-dATP by nick translation to the same specific activity and $5 \times 10^{6} \mathrm{cpm}$ per milliliter of this probe was used for the hybridization. The specificity of the hybridization was also controlled by adding to the hybridization cocktail a 100-fold molar excess of ARPP-16/19, ARPP-21, or DARPP32 cDNA fragments, nick translated in the presence of nonradioactive nuclcotides. Following hybridization, the sections were rinsed five times for $15 \mathrm{~min}$ in $1 \times \mathrm{SSC}$ at $55^{\circ} \mathrm{C}$. Finally, the sections were rinsed in autoclaved water for $2 \mathrm{~min}$, and then dehydrated through a series of graded alcohol solutions and air dried. The tissue sections were subsequently exposed to $\mathrm{x}$-ray films ( $\beta$-max; Amersham) for 14-21 d.

Image analysis. Autoradiograms from sections hybridized to ${ }^{35} \mathrm{~S}$-labeled cDNA fragments encoding ARPP-16/19, ARPP-21, and DARPP32 were used for semiquantitative analysis. The optical density values were measured using an MTI CCD high-resolution video camera connected to a Macintosh computer using the program IMAGE 1.31 (NIH). The highest optical density value for ARPP-16/19, ARPP-21, and DARPP-32, respectively was arbitrarily set to 100 and background was set as 0 in a linear scale.

\section{Results}

\section{Sequence analysis of human cDNA clones}

Two human striatal cDNA libraries were screened. It was determined by differential Southern hybridization with rat and bovine probes, and sequence analysis, that single or composite clones had been obtained containing 5 '-untranslated sequence, 
MSEOGDLNOAIAEEGGTEOETATPENGIVKSESIDEEEKLEIORRTEAONOERRKSKSGA ..P. . .S.T.V. . . . . . . . .VI . . . . . . . . . . . . . . .



\section{9}

GKGKLTRSLAVCEESSAR PGGESLQDQTL

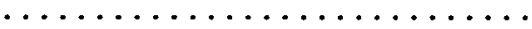

$\ldots \ldots$

C. ARPP -16
MEDKVTSPEKAEEAKLKARYPHLGQKPGGSDFLRKRLQKGQKYFDSGDYNMAKAKMKNKO

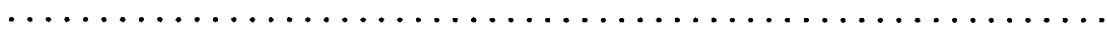

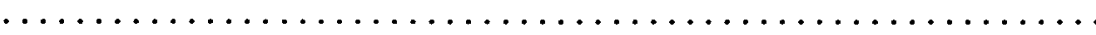

96

ETGDHIPTPODLPQRKPSLVASKLAG

$\ldots \ldots$. . . . . . . . . . . . .
Figure 1. Deduced amino acid sequences (in single-letter amino acid code) of human $(H)$, bovine $(B)$, and rat $(R)$ DARPP-32, ARPP-21 and ARPP-16. - signifies identity; - signifies deletion. the translation start site and a polyA tail (data not shown). For DARPP-32, the coding sequence was derived from two overlapping clones (HG1, HZ8), and for ARPP-21, the entire coding sequence was confirmed from three separate clones (HA3.1, HA5.1, and HA5.3). ARPP-16 and ARPP-19 are two separate proteins that differ only in their amino terminus (Horiuchi et al., 1990) and appear to be derived via alternate splicing from a single gene (A. Horiuchi and P. Greengard, unpublished observations). The probe derived from the common coding region therefore hybridized with the mRNAs for both ARPP-16 and ARPP-19. For ARPP-16, the coding sequence was confirmed from four separate clones (HK. 1-7.4). In addition, an ARPP$19 \mathrm{cDNA}$ was isolated that was identical except for the $5^{\prime}$ end, which was identical to the rat ARPP-19 cDNA (data not shown).

The probes used for the in situ hybridization analysis in this report were derived primarily, but not exclusively, from the coding regions from the clones described above. Degrees of conservation of sequence at the nucleotide and deduced amino acid level among human, bovine, and rat, calculated with reference to human, are shown in Table 1. The three scquences are highly conserved at both levels, but there are notable dif- ferences (Fig. 1). ARPP-16 is almost 100\% identical over its entire length. DARPP-32 is particularly conserved through the amino terminus, which represents the active portion of the protein (Hemmings et al., 1984). ARPP-21 is highly conserved in the carboxy half, which contains the phosphorylated serine (res-

Table 1. Percentage identity of the nucleotide coding sequences and deduced amino acid sequences between human, bovine, and rat for DARPP-32, ARPP-21, and ARPP-16

\begin{tabular}{llll} 
& Human & Bovine & Rat \\
\hline Nucleotide coding sequences & & & \\
DARPP-32 & 100 & 89 & 85 \\
ARPP-21 & 100 & 89 & 82 \\
ARPP-16 & 100 & 95 & 92 \\
Deduced amino acid sequences & & \\
DARPP-32 & 100 & 88 & 85 \\
ARPP-21 & 100 & 89 & 89 \\
ARPP-16 & 100 & 99 & 99 \\
\hline
\end{tabular}


A



B

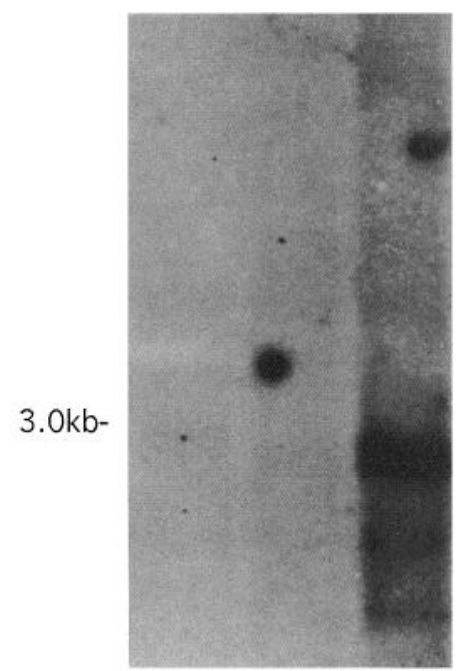

C



Figure 2. Northern blot analysis of $15 \mu \mathrm{g}$ of polyadenylated RNA from (left to right) Brodmann area 4, Brodmann area 9, and the head of the caudate nucleus, probed with partial length cDNAs for human ARPP16/19 (A), ARPP-21 $(B)$, and DARPP-32 (C).

idue 56 in the human protein), and may represent the active portion of the molecule. For both DARPP-32 and ARPP-21, the majority of the amino acid changes that do occur, between either two or three species, are conservative. Partial sequence analysis of the $5^{\prime}$ - and 3 '-noncoding regions was also performed, and interestingly, areas of up to $90 \%$ homology between species were detected (data not shown). There was no significant homology between the untranslated sequences of the clones of DARPP-32, ARPP-21, and ARPP-16.

\section{Northern blot analysis}

Northern blot analysis of polyadenylated RNA from caudate nucleus and the frontal cortex (Brodmann areas B4 and B9) was performed to determine the size of the mRNAs for DARPP32 , ARPP-16/19, and ARPP-21. In addition, these studies demonstrated the integrity of the mRNA from these specimens. Consistent with previous analyses in bovine and rat (Kurihara et al., 1988, 1989; Ehrlich et al., 1990; Horiuchi et al., 1990; Horiuchi and Greengard, unpublished observations), the major DARPP-32, ARPP-16/19, and ARPP-21 bands were 2.1, 1.7, and 3.0 kilobases, respectively (Fig. 2). Also consistent with previous studies, it is likely that the major bands in each case represent alternative forms of each of the mRNAs, due either to polyadenylation or to differences in the $5^{\prime}$-untranslated ends. There is no evidence from any of the three species for alternative splicing within the coding regions of the three proteins, except for the relationship between ARPP-16 and - 19 discussed above. For DARPP-32 and ARPP-21, there is clearly more mRNA in the caudate than in the cortex, where ARPP-21 is barely detectable (in contrast to what was previously seen in bovine and rat). In assessing the relative quantities of mRNA hybridizing to the ARPP-16 probe, it must again be noted that this probe also hybridizes to ARPP-19 mRNA, which is homogeneously distributed, and not neuron specific (Girault et al., 1990).

\section{Specificity of the in situ hybridization}

Whole hemisphere horizontal sections of eight representative levels from two human brains were hybridized to ARPP-16/19, ARPP-21, or DARPP-32 cDNA probes. The results showed varying intensities of labeling over specific brain regions with all three probes at all levels analyzed. The specificity of the in situ hybridization was assessed using different controls. No labeling was seen in any areas of the human brain after an adjacent section had been hybridized to a ${ }^{35}$ S-dATP-labeled pBSKS plasmid without a cDNA insert (Fig. $3 b$ ). The addition of a 100fold molar excess of nick-translated unlabeled ARPP-16/19 cDNA fragment to the ${ }^{35} \mathrm{~S}$-dATP labeled ARPP-16/19 cDNA probe abolished the labeling completely in all regions (Fig. $3 d$ ). The labeling seen with the ARPP-21 and DARPP-32 cDNA probes, respectively, was likewise abolished by the addition of an excess of the unlabeled probe (data not shown). Moreover, though all probes labeled cells in caudate nucleus, putamen, and regions of neocortex, the relative intensity of labeling in these regions was clearly different for the three probes (compare, e.g., Fig. $4 a-c)$.

\footnotetext{
Figure 3. Contact copies of autoradiograms. $a$, hybridization of a whole hemisphere section at the level of $71 \mathrm{~mm}$ ventral of vertex in the right brain hemisphere using a ${ }^{35}$ S-dATP-labeled cDNA fragment specific for ARPP-16/19 mRNA. Note the strong labeling over neocortex, caput caudatus, cauda caudatus, and putamen. $b$, Hybridization of a radiolabeled pBSKS plasmid DNA to a section at the level of 71 mm ventral of vertex in the right brain hemisphere. Note the absence of specific hybridization in all regions. $c$, Radiolabeled ARPP-16/19 cDNA hybridized to a section at the level of $91 \mathrm{~mm}$ ventral of vertex in the right side hemisphere. Note the labeling over the amygdaloid complex, neocortex, hippocampus, and cerebellar cortex. $d$, Hybridization of a section adjacent to the one shown in $c$ with the radiolabeled ARPP-16/19 cDNA fragment in the presence of a 100-fold excess of the same DNA fragment nick translated in the absence of labeled nucleotides. Note the displacement of specific labeling from all regions. Amy, amygdaloid complex; Cap, caput caudatus; Cau, cauda caudatus; Cbm, cerebellar cortex; Hip, hippocampus; Put, putamen; Th, thalamus. Numbers shown refer to Brodmann areas in the adjacent neocortex.
} 


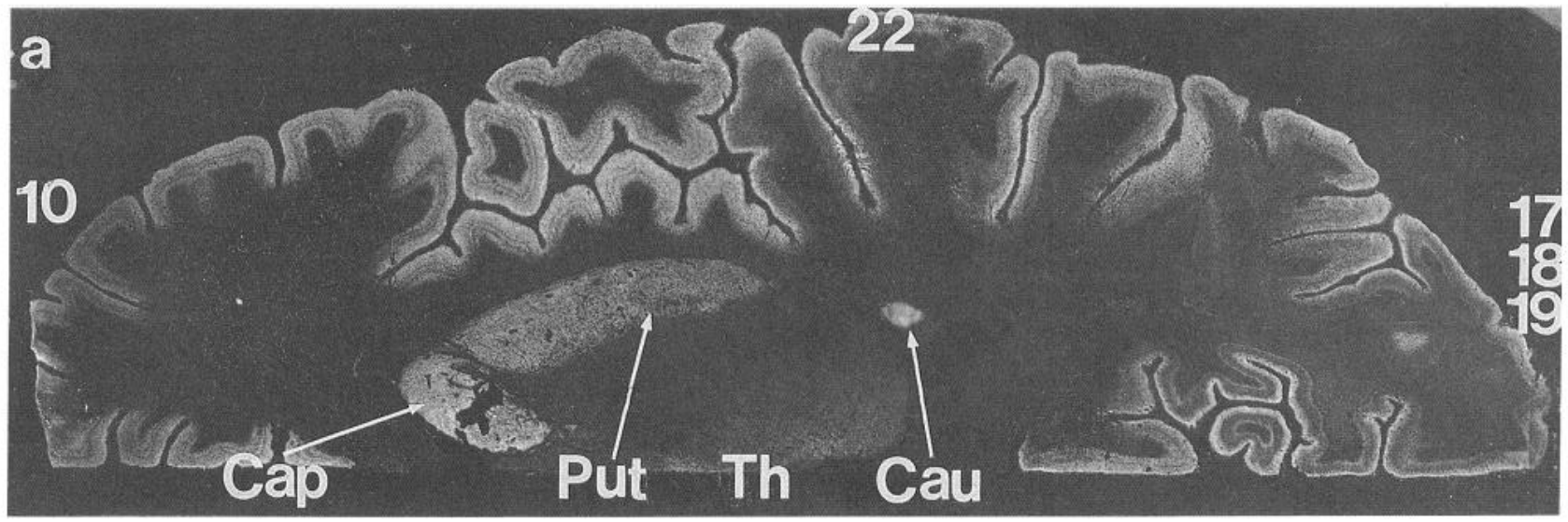

b

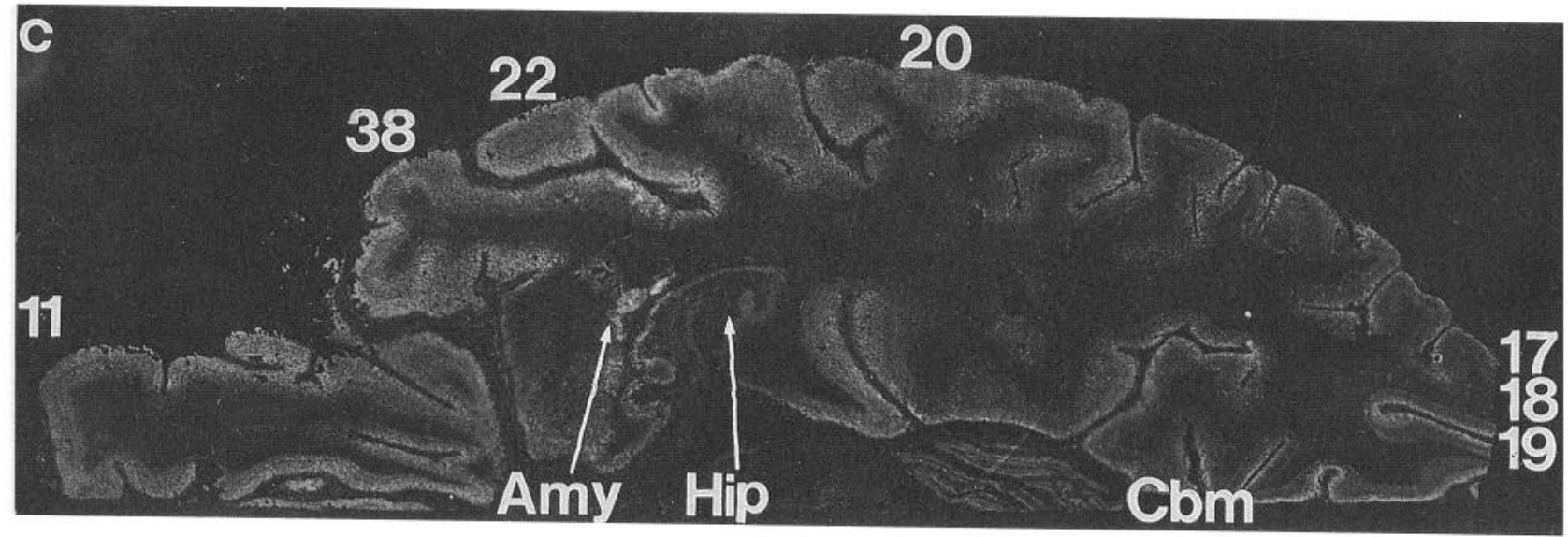

d 



Figure 4. $a$, ARPP-16/19; $b$, ARPP-21; and $c$, DARPP-32 mRNA expression in postmortem human brain, $71 \mathrm{~mm}$ below vertex in the right hemisphere. The boxed area is shown in higher magnification in Figure 10 (top). Abbreviations are as in Figure 3.

\section{Regional distribution of ARPP-16/19 mRNA}

The intensity of labeling in different layers of the neocortex was not similar in all Brodmann areas. In Brodmann areas 17, 18, and 19 , and in occipital cortex the intensity of labeling varied the most, with highest intensity in superficial layers, modest intensity in deeper layers and the lowest labeling in the intermediate layers (Figs. $4 a, 5 a, 6 a, 7,8 a$ ). In Brodmann area 10 and in insular cortex, high intensity of labeling could be seen in a superficial, an intermediate, and a deeper layer. These three layers were separated by two layers that had little or no labeling (Figs. $4 a, 5 a, 6 a, 7$ ).

Both the head and tail of the caudate nucleus as well as the putamen and nucleus accumbens contained high levels of ARPP16/19 mRNA (Figs. $4 a, 5 a, 6 a$ ). The labeling appeared to be higher in nucleus caudatus than in putamen. The labeling intensity for ARPP-16/19 mRNA was similar in neocortex and the basal ganglia structures (Figs. $4 a, 5 a, 6 a$ ). Lower levels were 

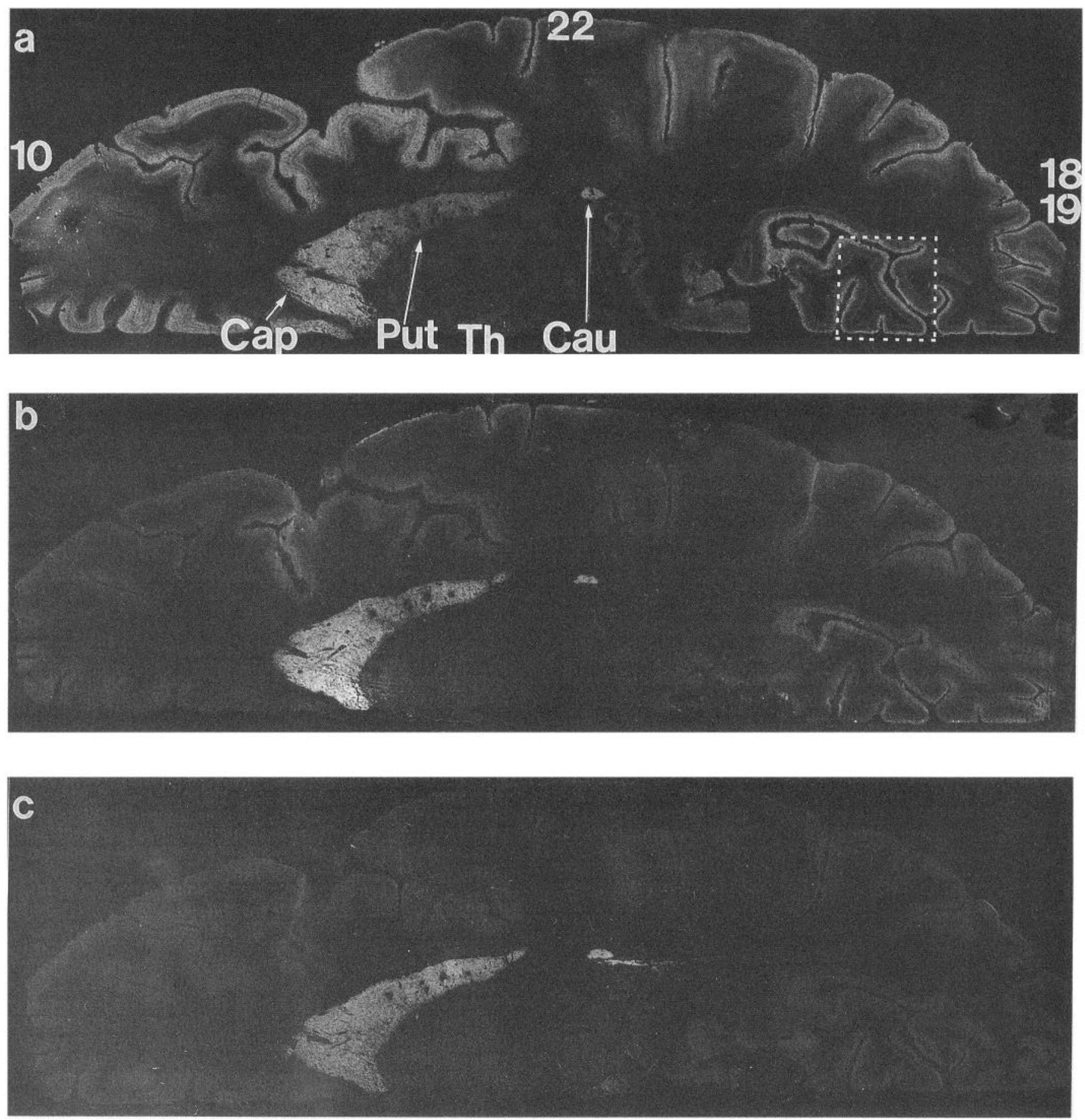

Figure 5. $a$, ARPP-16/19; $b$, ARPP-21; $c$, DARPP-32 mRNA expression in postmortem human brain, $78 \mathrm{~mm}$ below vertex in the right hemisphere. The boxed area is shown in higher magnification in Figure 10 middle panel. Abbreviations are as in Figure 3.

found in the choroid plexus, $\mathrm{CA1}-\mathrm{CA} 3$, and dentate gyrus regions in the hippocampus (Figs. $6 a, 7,8 a$ ) and subnuclei of the amygdaloid complex (Figs. $6 a, 8 a$ ).

The most ventral brain levels examined showed a distinct distribution of ARPP-16/19 mRNA. The labeling in neocortical Brodmann areas 18 and 19 had a sharp and distinct variation in intensity of hybridization in the different neocortical layers, with the highest labeling in superficial layers, modest levels in deeper layers, and low levels in the intermediate layers. Brod- mann areas 20, 22, and 38 showed a more even labeling over the neocortical layers. In Brodmann area 11, varying intensities of labeling were seen in three layers that were separated by layers that had very low or no labeling (Figs. $8 a, 9 a$ ). At the level of $97 \mathrm{~mm}$ ventral to vertex the labeling intensity in the cerebellar cortex was the same as in the neocortex. At more ventral levels the labeling intensity over cerebellar cortex was even higher than over neocortex (Fig. 9a). The distribution of ARPP-16/19 mRNA 

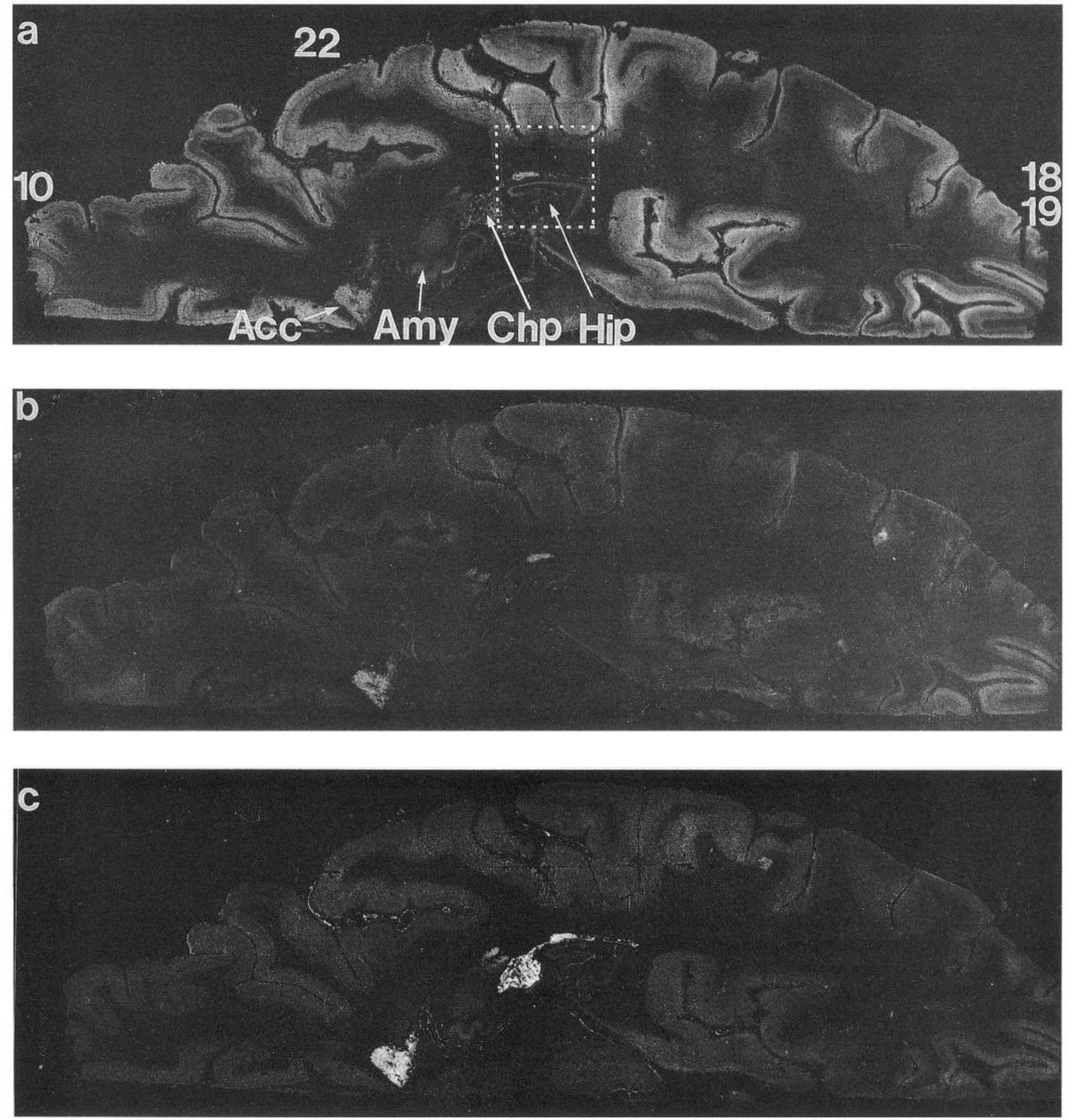

Figure 6. $a$, ARPP-16/19; $b$, ARPP-21; and $c$, DARPP-32 mRNA expression in postmortem human brain $88 \mathrm{~mm}$ below vertex in the right hemisphere. $A c c$, nucleus accumbens; $C h P$, choroid plexus; other abbreviations are as in Figure 3.

showed a distinct deeper lamination in the cerebellar cortex, which appears to correspond to the granular layer.

\section{Regional distribution of ARPP-21 $\mathrm{mRNA}$}

The labeling pattern for ARPP- 21 mRNA in the most apical tissue levels was similar to the pattern seen for ARPP-16/19 mRNA. In the neocortical layers the labeling was highest in superficial layers and lower in deep layers (Figs. $4 b, 5 b, 6 b, 7$ ).
For ARPP-21 mRNA the intensity of hybridization over caudate nucleus and putamen was higher than the labeling over neocortical regions (Figs. $4 b, 5 b$ ). In contrast to ARPP-16/19 mRNA, the level of ARPP-21 mRNA appeared to be higher in caput caudatus than in putamen and cauda caudatus (Figs. $4 b$, $5 b$ ). Nucleus accumbens was the most intensely labeled neuronal area in the brain for ARPP-21 mRNA at the level of $88 \mathrm{~mm}$ ventral of vertex (Fig. $6 b$ ). Compared to nucleus accumbens, 


\section{ARPP-16/19}
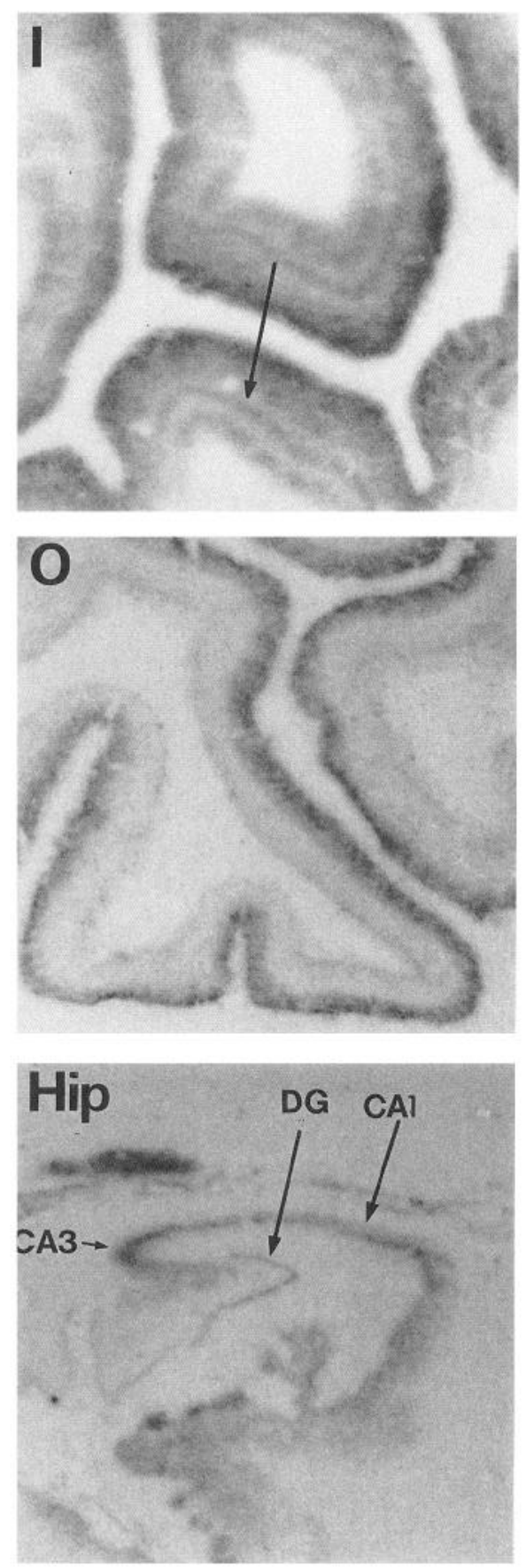

ARPP-21
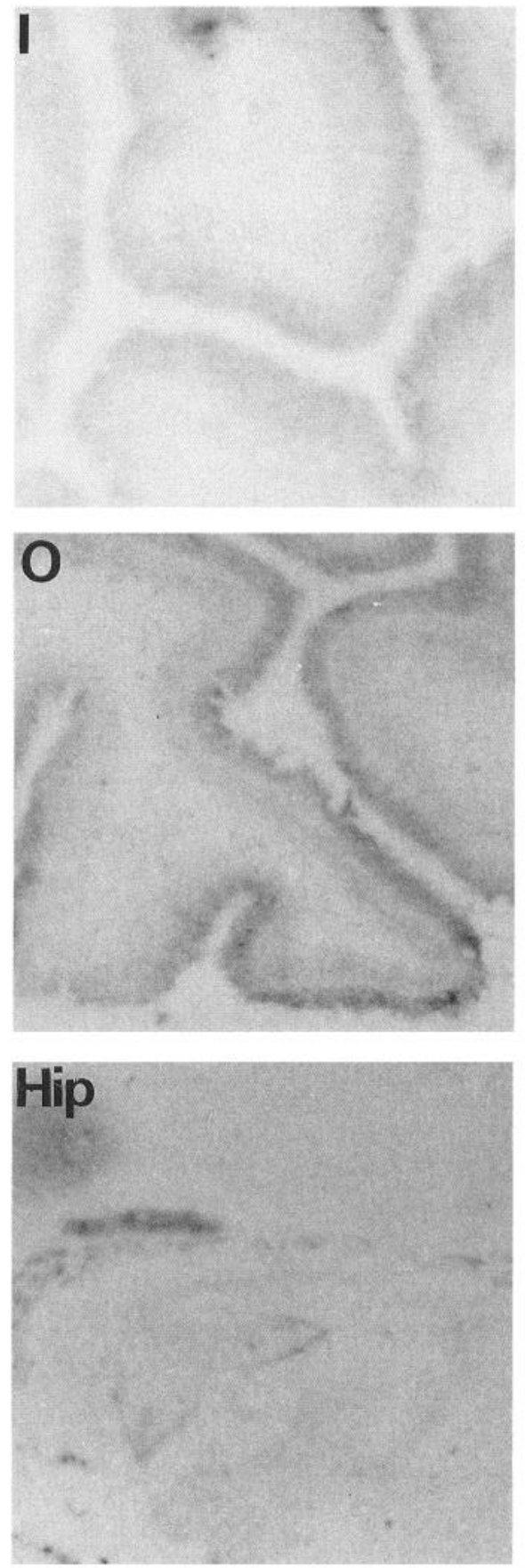

DARPP-32


Figure 7. High-magnification bright-field autoradiograms showing insular and occipital cortex and the hippocampal formation after hybridization to the respective phosphoprotein probe. Arrow in the upper panel is pointing at the insular cortex. I, insular cortex; Hip, hippocampus; $O$, occipital cortex; $C A 1, C A 3$, pyramidal cell layers of the hippocampus; $D G$, dentate gyrus.

low levels were detected in the dentate gyrus and the CAl and CA3 regions of the hippocampal formation (Figs. $6 b, 7,8 b$ ). Low levels of ARPP-21 mRNA were also present in different subnuclei of the amygdaloid complex and in the choroid plexus (Figs. $6 b, 7,8 b$ ).

A relatively intense hybridization for ARPP-21 mRNA was seen within the cerebellar cortex, where the distribution of cells expressing ARPP-21 mRNA showed a deeper lamination, possibly corresponding to the granular layer (Figs. $8 b, 9 b$ ).

\section{Regional distribution of DARPP-32 mRNA}

A low labeling for DARPP-32 mRNA was seen in all neocortical layers with the strongest hybridization in the superficial layers (Figs. $4 c, 5 c, 6 c, 7,8 c$ ). An intense labeling was seen over both the head and tail of the caudate nucleus as well as the putamen and nucleus accumbens (Fig. $4 c, 5 c, 6 c, 7$ ). For DARPP-32 mRNA the intensity of hybridization over caudate nucleus and putamen was markedly higher than the labeling over neocortical 

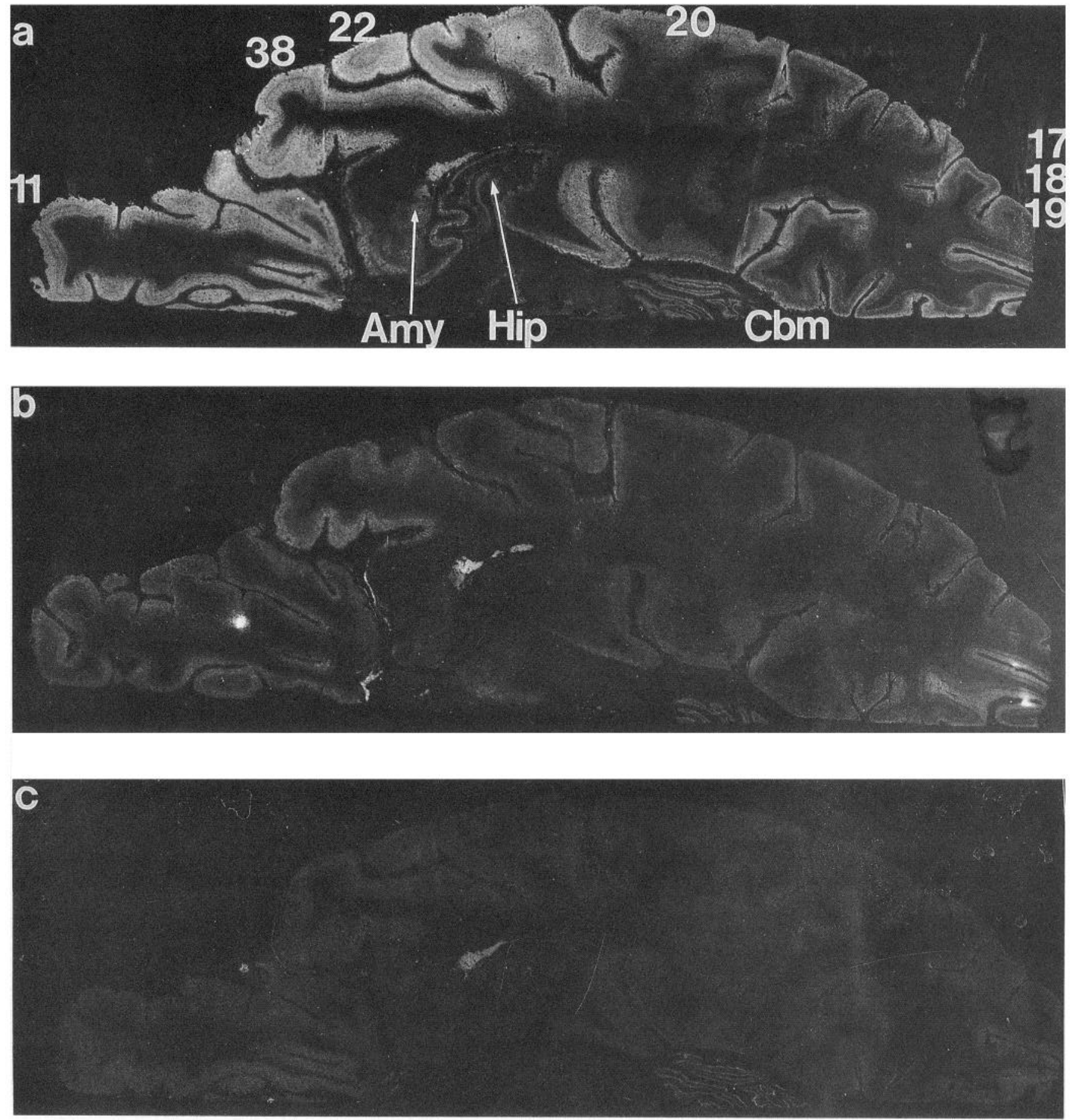

Figure 8. $a$, ARPP-16/19; $b$, ARPP-21; and $c$, DARPP-32 mRNA expression in postmortem human brain $91 \mathrm{~mm}$ below vertex in the right hemisphere. Abbreviations are as in Figure 3.

regions. The level of DARPP-32 mRNA was higher in cauda caudatus compared to caput caudatus. Similar to ARPP-21 mRNA, the nucleus accumbens was the most intensely labeled neuronal area in the brain at the level of $88 \mathrm{~mm}$ ventral of vertex (Fig. 6c). DARPP-32 mRNA was found in the choroid plexus at labeling intensities comparable to those seen in the basal ganglia (Fig. 6c). Lower levels of DARPP-32 mRNA were detected in the dentate gyrus, the CAl and CA3 regions in the hippocampus, and different subnuclei of the amygdaloid complex (Figs. 6c, 7, 8c).
DARPP-32 mRNA was also detected at relatively high levels within the cerebellar cortex (Figs. $8 c, 9 c$,) where it was concentrated in a narrow deep layer that appeared to correspond to the Purkinje cell layer.

\section{Image analysis}

The levels of mRNA for the three phosphoproteins were also quantified using pseudocolored images of autoradiograms. At the level of $71 \mathrm{~mm}$ ventral of vertex ARPP-16/19 labeling showed similar optical density values over putamen and the deeper 

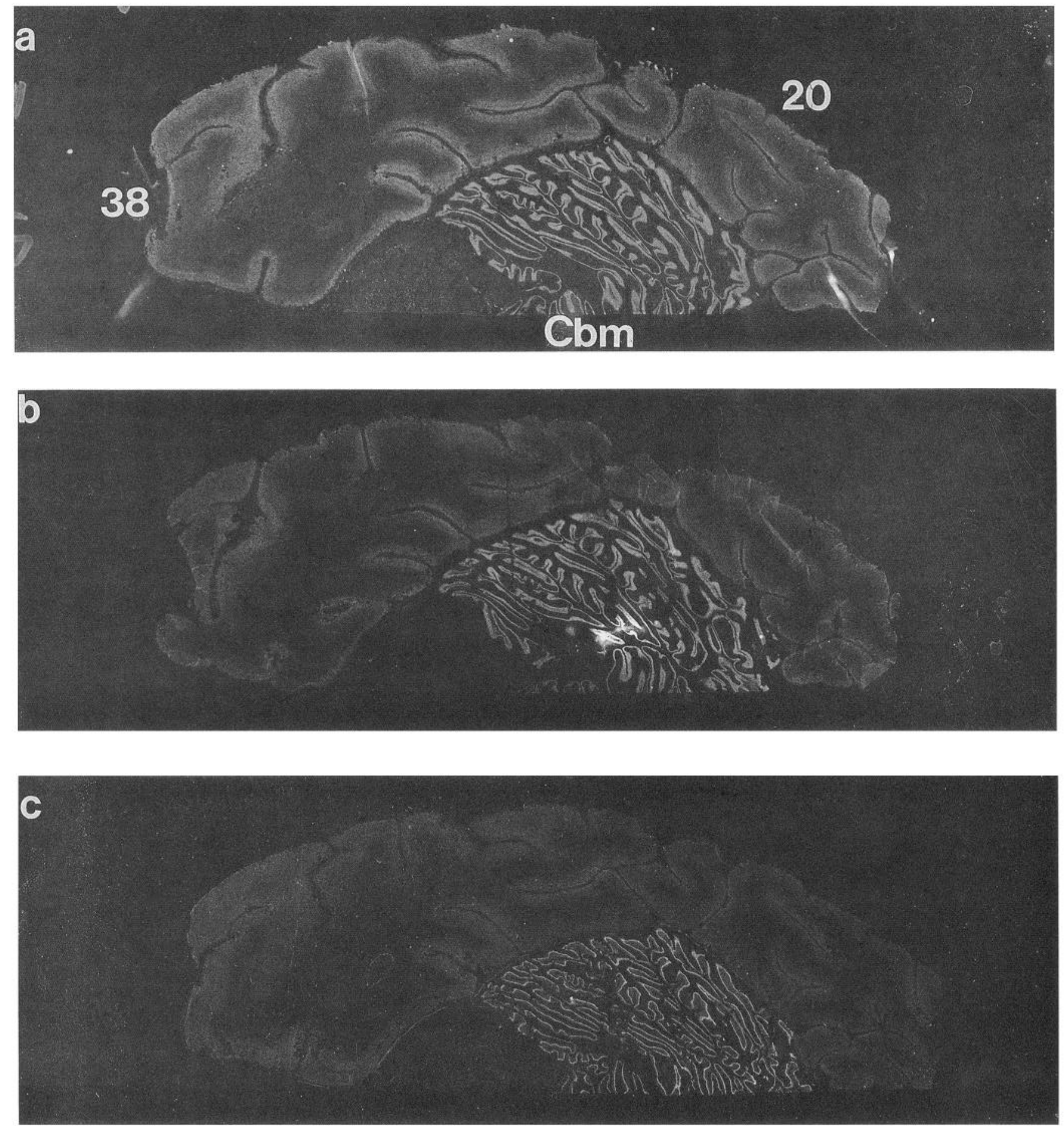

Figure 9. $a$, ARPP-16/19; $b$, ARPP-21; and $c$, DARPP-32 mRNA expression in post mortem human brain $104 \mathrm{~mm}$ below vertex in the right hemisphere. $\mathrm{Cbm}$, cerebellar cortex.

neocortical layers (Fig. 10, top). Intermediate optical density values were obtained over superficial cortical layers and lateral caput and cauda caudate nucleus. The highest optical density value was found over medial parts of caput caudate nucleus. ARPP-21 labeling showed low optical density values in neocortex, and intermediate values in putamen, cauda caudatus, and lateral parts of caput caudatus. The highest level was found in medial caput caudatus (Fig. 10, middle). DARPP-32 labeling showed low optical density values in neocortex, intermediate values in putamen, and the highest values in caput and cauda caudatus and the choroid plexus (Fig. 10, bottom).

\section{Emulsion autoradiograms}

Photographic emulsions of $14-\mu \mathrm{m}$-thick block sections from the caudate nucleus of one brain hybridized to the three phosphoprotein cDNA probes revealed labeling over most of the medium size neurons in the caudate nucleus with all three probes (Fig. 11). 



Figure 10. Semiquantitative pseudocolored images of autoradiograms after hybridization to whole brain tissue hemisphere sections at the level of $71 \mathrm{~mm}$ ventral of vertex. The hybridizations were performed using ${ }^{35} \mathrm{~S}$ labeled cDNA fragments encoding ARPP-16/19 (top), ARPP-21 (middle), DARPP-32 (bottom).

\section{Discussion}

In this report we have analyzed the human cDNAs for the cAMP-regulated phosphoproteins DARPP-32, ARPP-21, and ARPP-16 to determine their sequences and to identify useful probes for the study of mRNA expression in the human postmortem brain. The high conservation of the nucleotide and deduced amino acid sequences of these proteins, particularly within what is likely to be the functional domains, implies conservation of function as well.

Both Northern blots and in situ hybridization were used to study the regional distribution of mRNAs encoding ARPP-16, ARPP-19, ARPP-21, and DARPP-32 in postmortem human brain. The use of whole hemisphere sections from the human brain for the in situ hybridization allowed a detailed examination of the regional distribution of these mRNAs. The postmortem time varied from 3 to $22 \mathrm{hr}$ in the three subjects in this study, but no correlation was seen between different postmortem times and the intensity and specificity of the hybridization. However, it cannot be excluded that the length of postmortem time and other conditions such as temperature, humidity, or cause of death can affect mRNA stability in postmortem tissue. It might even be that some brain regions are more vulnerable to mRNA degradation than others, which therefore could affect the intensity of the hybridization signal in a region-specific manner. Several criteria were used to assess the specificity of the in situ hybridization. These included (1) the abolition of the hybridization by addition of excess, unlabeled probe and (2) the lack of hybridization over any brain region using a labeled pBSKS plasmid not containing a cDNA insert; (3) the three radiolabeled cDNA fragments showed similar hybridization patterns in basal ganglia structures and neocortex but the relative intensity of labeling varied markedly in these structures. Thus, the different relative distributions observed for the three mRNAs in these 
structures supports the specificity of hybridization. (4) Parts of the in situ hybridization results, that is, the higher labeling for ARPP-21 and DARPP-32 mRNAs in basal ganglia structures than in neocortex, with more even labeling intensities over these structures for ARPP-16/19 mRNA, were confirmed by Northern blot analyses. (5) Emulsion autoradiograms revealed that the intense labeling seen with all three probes over the caudate nucleus on $x$-ray autoradiograms corresponded to labeling over individual neurons of the medium-size type.

\section{Expression of ARPP-16/19 $\mathrm{mRNA}$}

In situ hybridization revealed high levels of ARPP-16/19 mRNA in caudate nucleus, putamen, nucleus accumbens, neocortex, and a deep layer of cerebellar cortex. Lower levels were seen in the amygdaloid complex, hippocampus, and the choroid plexus. The distribution of ARPP-16/19 mRNA agrees with previous findings of high levels of ARPP-16 and ARPP-19 in the caudate nucleus and regions of the neocortex and the cerebellar cortex in rat and monkey (Girault et al., 1990). The ARPP-16/19specific cDNA probe revealed a more precise distribution pattern, particularly in the neocortical regions, where the labeling pattern in different layers of neocortex was striking, but a more detailed microscopic examination of the tissue was difficult due to the thickness of the sections. The laminar distribution of ARPP-16/19 mRNA in human neocortical brain regions and the regional distribution in subcortical brain regions such as caudate nucleus, putamen, nucleus accumbens, and hippocampus closely overlaps with dopamine D1 receptor binding sites in the primate and human brain. However, the strong ARPP$16 / 19$ mRNA labeling in cerebellar cortex and choroid plexus does not match with previously described dopamine $\mathrm{D}_{1}$ receptor binding sites (Hall et al., 1988; Cortés et al., 1989; Richfield et al., 1989).

\section{Expression of $A R P P-21 \mathrm{mRNA}$}

In situ hybridization showed an intense labeling for ARPP-21 mRNA in nucleus caudatus and cerebellar cortex with only low labeling over neocortex. Similar to ARPP-16/19 mRNA, the pattern of ARPP-21 mRNA labeling in the neocortex showed a laminar distribution with the highest level in superficial layers. The overall intensity of labeling in these layers was, however, significantly lower than for ARPP-16/19 mRNA. The less abundant expression of ARPP-21 mRNA in the neocortex compared to basal ganglia structures in the human brain agrees with findings of lower levels of ARPP-21 in neocortex than in nucleus caudatus and putamen in the rat (Girault et al., 1990; Ehrlich and Greengard, 1991).

\section{Expression of DARPP-32 $m R N A$}

The relative levels of DARPP-32 mRNA in the basal ganglia structures and neocortex were similar to the relative distribution of ARPP-21 mRNA. However, differences in the labeling pattern were seen between the two probes. In particular, the choroid plexus was labeled for DARPP-32 with the same intensity as the labeling over nucleus caudatus whereas a much higher labeling of nucleus caudatus than of choroid plexus was found for ARPP-21 mRNA. Furthermore, in the cerebellar cortex a distinct layer of DARPP-32 mRNA labeled cells was found that probably corresponds to cells in the Purkinje layer. In contrast, ARPP-21 mRNA was more abundant in a deep layer in the cerebellar cortex.
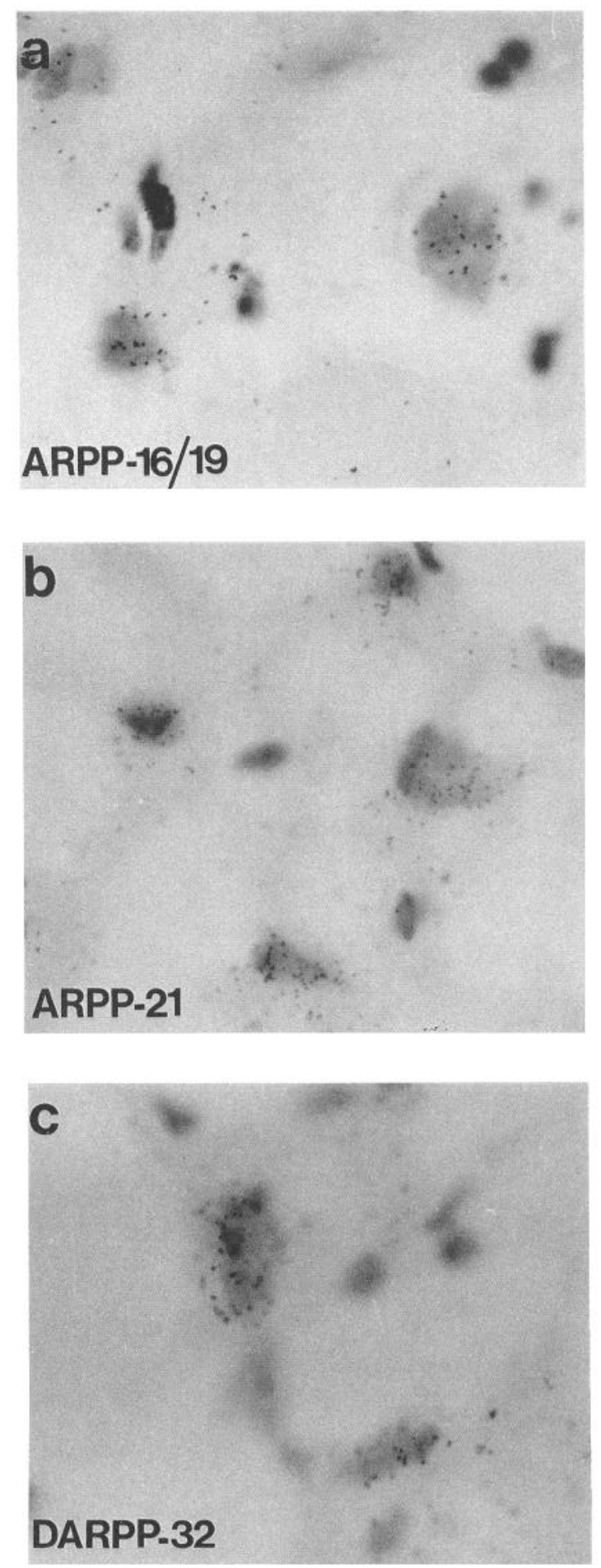

Figure 11. Bright-field emulsion autoradiograms of $14-\mu \mathrm{m}$-thick block sections from caudate nucleus hybridized to ARPP16/19 (a), ARPP-21 (b), or DARPP-32 (c) mRNA-specific probes.

\section{Functional implications}

The regional distributions of mRNAs encoding the three phosphoproteins overlap to a large extent with $D_{1}$ receptor binding sites in the primate and human brain (Hall et al., 1988; Cortés et al., 1989; Richfield et al., 1989). Moreover, most of these regions express high levels of DARPP-32 mRNAs. The results support the hypothesis that dopamine, via the adenylyl cyclase 
system, is likely to regulate the function of these proteins in these regions. Notable exceptions are the cerebellar cortex and choroid plexus. It has recently been shown that phosphorylation of DARPP-32 in the choroid plexus of the rat is regulated by norepinephrine and 5-HT (Snyder et al., 1992), and that DARPP32 therefore may play a role in the control of secretion of cerebrospinal fluid. An increase in phosphorylation of striatal ARPP-21, ARPP-19, and ARPP-16 by vasoactive intestinal peptide in vitro has also been demonstrated (Girault et al., 1988). Anatomical correlations with receptors for other neurotransmitters and peptides should yield further clues as to the function of these proteins. In this regard, it will be interesting to determine the nature of substances regulating the three phosphoproteins in the cerebellar cortex, which in the human contains surprisingly high mRNA levels.

The results of the present in situ hybridization on whole hemisphere sections demonstrate that this technique can be used to obtain detailed information on the regional distribution of different mRNAs in the human brain. Combincd with appropriate hybridization controls, such as the abolition of the labeling by an excess of unlabeled probe, this technique should be useful for future studies on the regional distribution of mRNAs encoding gene products with a role in the CNS. In fact, recent studies have provided a detailed map at certain levels of cells expressing the neuropeptide cholecystokinin (Lindefors et al., 1991, 1993). An interesting fulure application of this technique includes comparative mapping of tissues from various neuropsychiatric clinical conditions and healthy human brain in order to assess differences in mRNA and its possible role in pathophysiological mechanisms for such disorders.

\section{References}

Cortés R, Camps M, Gueye B, Probst A, Palacios JM (1989) Dopamine receptors in human brain: autoradiographic distribution of DI and D2 sites in Parkinson syndrome of different etiology. Brain Res 483:30-38.

Ehrlich ME, Greengard P (1991) Characterisation of rat ARPP-21 mRNA: sequence analysis, tissue distribution, and regulation. J Neurochem 57:1985-1991.

Ehrlich ME, Kurihara T, Greengard P (1990) Rat DARPP-32: cloning, sequencing, and characterization of the cDNA. J Mol Neurosci 2:110.

Fonnum F, Storm-Mathiesen J, Divac I (1981) Biochemical evidence for glutamate as neurotransmitter in corticostriatal and corticothalamic fibers in rat brain. Neuroscience 6:863-873.

Girault J-A, Shalaby IA, Rosen NL, Greengard P (1988) Regulation by CAMP and vasoactive intestinal peptide of phosphorylation of specific proteins in striatal cell culture. Proc Natl Acad Sci USA 85: 7790-7794.

Girault J-A, Waalas SI, Hemmings HJ, Greengard P (1990a) ARPP21, a cAMP-regulated phosphoprotein enriched in dopamine-innervated brain regions: tissue distribution and regulation of phosphorylation in the rat. Neuroscience 37:317-325.

Girault J-A, Horiuchi A, Gustafson EL, Rosen NL, Greengard P (1990b) Differential expression of ARPP-16 and ARPP-19, two highly related
cAMP-regulated phosphoproteins, one of which is specifically associated with dopamine-innervated brain regions. J Neurosci 10:11241133.

Greengard P (1987) Neuronal phosphoproteins. Mediators of signal transduction. Mol Neurobiol 1:81-119.

Hall H, Farde L, Sedvall G (1988) Human dopamine receptor subtypes - in vitro binding analysis using ${ }^{3} \mathrm{H}-\mathrm{SCH} 23390$ and ${ }^{3} \mathrm{H}-$ raclopride. J Neural Transm 73:7-21.

Halpain S, Girault J-A, Greengard P (1990) Activation of NMDA receptors induces dephosphorylation of DAR PP-32 in rat striatal slices. Nature 343:369-372.

Hemmings HC Jr, Greengard P (1989) ARPP-21, a cyclic AMP regulated phosphoprotein enriched in dopamine-innervated brain regions. I. Purification and characterization of the protein from bovine caudate nucleus. J Neurosci 9:851-864.

Hemmings HC Jr, Greengard P, Lim Tung HY, Cohen P (1984) DARPP-32, a dopamine-regulated neuronal phosphoprotein, is a potent inhibitor of protein phosphatase-1. Nature 310:503-505.

Horiuchi A, Williams KR, Kurihara T, Nairn AC, Greengard P (1990) Purification and cDNA cloning of ARPP-16, a cAMP-regulated phosphoprotein enriched in basal ganglia, and of a related phosphoprotein, ARPP-19. J Biol Chem 265:9476-9484.

Kurihara T, Lewis RM, Eisler J, Greengard P (1988) Cloning of cDNA for DARPP-32, a dopamine and cyclic AMP-regulated neuronal phosphoprotein. J Neurosci 8:508-517.

Kurihara T, Ehrlich ME, Horiuchi J, Nasu T, Greengard P (1989) ARPP-21, a cyclic AMP-regulated phosphoprotein enriched in dopamine-innervated brain regions. II. Molecular cloning and nucleotide sequence. J Neurosci 9:3638-3544.

Lindefors N, Brené S, Kopp J, Lindén A, Brodin E, Sedvall G, Persson $H$ (1991) Distribution of cholecystokinin mRNA and peptides in the human brain. Neuroscience 42:813-821.

Lindefors N, Lindén A, Brené S, Sedvall G, Persson H (1993) CCK peptides and mRNA in the human brain. Prog Neurobiol 40:671690.

Ouimet CC, Greengard P (1990) Distribution of DARPP-32 in the basal ganglia: an electron microscopic study. J Neurocytol 19:39-52.

Ouimet CC, Miller PE, Hemmings HC Jr, Walaas SI, Greengard P (1984) DARPP-32, a dopamine and adenosine $3^{\prime}: 5^{\prime}$-monophosphate-regulated phosphoprotein enriched in dopamine-innervated brain regions. $J$ Neurosci 4:111-124.

Ouimet CC, Hemmings HC Jr, Greengard P (1989) ARPP-21, a cyclic AMP-regulated phosphoprotein enriched in dopamine-innervated brain regions. II. Immunocytochemical localization in the brain. J Neurosci 9:865-875.

Richfield EK, Young AB, Penney JB (1989) Comparative distribution of dopamine D-1 and D-2 receptors in the cerebral cortex of the rats, cats, and monkeys. J Comp Neurol 286:409-426.

Schalling M, Djurfeldt M, Hökfelt T, Ehrlich M, Kurihara T, Greengard P (1990) Distribution and cellular localization of DARPP-32 mRNA in the rat. Mol Brain Res 7:139-149.

Snyder G, Girault J-A, Chen JC, Czernik AJ, Kebabian JW, Nathanson JA, Greengard P (1992) Phosphorylation of DARPP-32 and protein phosphatase inhibitor-1 in choroid plexus: regulation by factors other than dopamine. J Neurosci 12:3071-3083.

Walaas SI, Greengard P (1984) DARPP-32, a dopamine and adenosine $3^{\prime}: 5^{\prime}$-monophosphate-regulated phosphoprotein enriched in dopamine innervated brain regions. I. Regional and cellular distribution in the rat brain. $\mathrm{J}$ Neurosci 4:84-98.

Walaas SI, Aswad DW, Greengard P (1983) A dopamine- and cyclic AMP regulated phosphoprotein enriched in dopamine-innervated brain regions. Nature 301:69-71. 